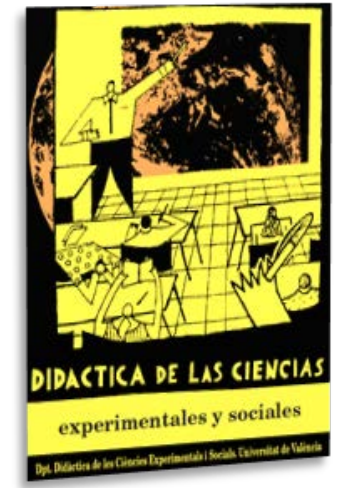

\title{
En busca de una didáctica de la sociología: aportes desde la educación secundaria brasileña
}

\author{
In search of a didactics of sociology: \\ contributions from Brazilian secondary \\ education
}

DOI: $10.7203 / D C E S .40 .17651$

\author{
Amurabi Oliveira \\ Universidad Federal de Santa Catarina (UFSC) (Brasil), amurabi_cs@hotmail.com \\ ORCID iD: http://orcid.org/0000-0002-7856-1196
}

\begin{abstract}
RESUMEN: Las ciencias sociales en la educación secundaria incorporan diferentes asignaturas escolares, como historia, geografía, psicología, sociología, etc. Sin embargo, el campo de la didáctica en las ciencias sociales se ha centrado en la didáctica de la historia y la geografía, como muestra la literatura especializada. Por otro lado, en países como Brasil, la discusión sobre la enseñanza de la sociología en la educación secundaria se ha expandido en los últimos años, señalando la necesidad de desarrollar una didáctica de la sociología. En este artículo, busco señalar los fundamentos de una didáctica de la sociología a partir de la realidad brasileña, sin embargo, dialogando con el campo más amplio de la didáctica en las ciencias sociales. Indico que la articulación entre temas, teorías y conceptos es fundamental para la enseñanza de la sociología en la educación secundaria, así como la necesidad de llevar a cabo una didáctica interdisciplinaria con la didáctica de las ciencias sociales.
\end{abstract}

Palabras Clave: enseñanza de sociología, didáctica de sociología, enseñanza de ciencias sociales, educación secundaria, interdisciplinariedad

ABSTRACT: Social sciences in secondary education incorporate different school subjects, such as history, geography, psychology, sociology, etc. However, the field of didactics of the social sciences has focused on the didactics of history and geography, as specialized literature shows. On the other hand, in countries such as Brazil, the discussion on the teaching of sociology in secondary education has expanded in recent years, pointing out the need to develop a didactics of sociology. In this article, I seek to point out the foundations of a didactics of sociology based on the Brazilian reality, however, dialoguing with the broader field of didactics in the social sciences. I indicate that the articulation between themes, theories and concepts is fundamental for the teaching of sociology in secondary education, as well as the need to carry out an interdisciplinary didactics with the didactics of the social sciences.

KEYWORDS: teaching of sociology, didactics of sociology, didactics of the social sciences, secondary education, interdisciplinarity

Fecha de recepción: junio de 2020

Fecha de aceptación: noviembre de 2020

Este artículo es el resultado de una pasantía post-doctoral en la Universidad Autónoma de Barcelona, financiada con una beca PVE-Junior CAPES y supervisada por el profesor Antoni Santisteban, a quien agradezco las contribuciones. 


\section{INTRODUCCIÓN}

La enseñanza de las ciencias sociales asume diferentes configuraciones en distintos países y regiones, centrándose muchas veces en la enseñanza de la geografía y la historia, el campo de las ciencias sociales también incorpora otras asignaturas como la antropología, la economía, la psicología y la sociología. También podemos verificar la existencia de asignaturas que combinan diferentes ciencias sociales, como en la enseñanza de ciencias socioeconómicas en Francia, o estudios sociales en países como Estados Unidos e Inglaterra. El punto en común que encontramos frecuentemente entre las distintas ciencias sociales en el currículum escolar es la formación para la ciudadanía, algo que está condicionado directamente por los cambios sociales y las reformas educativas (Oliveira y Santisteban, 2020).

En la literatura producida en países como España, la producción académica en didáctica de las ciencias sociales (DCS) se centra principalmente en el campo de la historia y la geografía, con poco espacio para la discusión sobre la didáctica de otras ciencias sociales (Gómez-Carrasco; López Facal y Rodríguez-Medin, 2019). En este sentido, la didáctica de la enseñanza de la sociología ha sido objeto de una reflexión aún incipiente en la literatura en castellano ${ }^{1}$.

Por otro lado, en Brasil la literatura sobre la enseñanza de la sociología en la educación secundaria se ha expandido, aumentando el número de tesis de maestría y doctorado sobre el tema (Bordart y Cigales, 2017) y artículos científicos publicados en revistas especializadas. (Oliveira y Melchioretto, 2020). Este crecimiento ha sido impulsado por la reintroducción nacional de la sociología en la educación secundaria en 2008, lo que ha permitido una creciente comunidad de investigadores sobre el tema, que realiza importantes intercambios en congresos y grupos de trabajos especializados. Además, es importante decir que mismo en el caso brasileño, no hay una reflexión específica para el desarrollo de una didáctica de la sociológica, pero hay un conjunto de experiencias en los últimos años, vividos por profesores de todo el país, así como una significativa producción de libros de texto.

Se observarse en los sistemas escolares de los países iberoamericanos la presencia dispersa de la sociología como asignatura de la educación secundaria. Esta ciencia social constituye una asignatura presente en el currículum de la educación secundaria obligatoria en Brasil, en el bachillerato en humanidades y ciencias sociales en Uruguay y algunas provincias en Argentina, además de ser una materia optativa en la educación secundaria postobligatoria (bachillerato de orientación en humanidades y/o ciencias sociales) de países como Ecuador, España y Portugal.

Considerando este escenario, es relevante desarrollar una reflexión inicial acerca de la didáctica de la enseñanza de sociología, insertándola en el campo más amplio de la DCS. En este artículo, busco desarrollar esta reflexión desde el caso brasileño, contextualizando la historia de esta asignatura en la educación secundaria brasileña, así como los avances y retrocesos que han tenido lugar en este país. Esa contextualización es especialmente relevante para los lectores que no conocen la realidad escolar de Brasil y la historia de esa asignatura en el currículum.

Mi objetivo, por lo tanto, es pensar en las bases de una didáctica de la sociología para la educación secundaria. Se trata de una síntesis inicial que presenta elementos para investigaciones futuras, que puedan orientar nuevas reflexiones para profesores brasileños que enseñan sociología en la educación secundaria y también profesores de otros países, especialmente de América Latina, España y Portugal.

\footnotetext{
${ }^{1}$ Una de las obras que destacan en este set es la colección organizada por Pipkin (2009) en Argentina.
} 


\section{LA ENSEÑANZA DE LA SOCIOLOGÍA EN LA ESCUELA BRASILEÑA}

La sociología se introdujo en el sistema escolar brasileño a fines del siglo XIX, cuando algunas escuelas crearon cátedras de sociología en la educación secundaria (Oliveira, 2013). En parte, este fenómeno puede explicarse por la fuerte recepción que tuvo el positivismo en Brasil en el siglo XIX, como lo demuestra el hecho de que la "Religión de la Humanidad", creada por Augusto Comte (1798-1857) tiene templos sólo en Francia y en Brasil. Esa recepción fue especialmente significativa en las Facultades de Derecho (Villas Bôas, 2006), de donde provienen muchos de los primeros profesores de sociología en Brasil, que recebían el título de licenciados en ciencias jurídicas y sociales (Engerroff y Cigales, 2017).

En medio de las reformas educativas iniciadas en la década de 1920, como Rocha Vaz (1925) y Francisco Campos (1931), la sociología comenzó a ganar espacio en el currículo escolar, convirtiéndose en una asignatura obligatoria de cursos complementarios, que permitieron a los estudiantes el ingreso a la educación superior. Los estudiantes que terminaron los cursos complementarios recibieron el título de Bachiller en Ciencias y Letras. Aunque las reformas educativas promovidas por los decretos federales no eran capaces de afectar directamente a los estados, sino sólo el Colégio Pedro II en el Distrito Federal, que era considerado un modelo para otras escuelas secundarias en otras partes de Brasil.

Cabe señalar que la transición del siglo XIX al XX estuvo marcada por numerosos eventos en la historia de Brasil, como la abolición de la esclavitud en 1888, el fin del imperio y el comienzo de la república en 1889, así como el fin de la Antigua República y el comienzo de la Nueva República en 1930. En medio de este conjunto de fenómenos, la introducción de la sociología, así como otras materias escolares en ese momento, representaba un esfuerzo por modernizar los planes de estudio escolares. No en vano, la sociología también se introdujo en las "Escuelas normales", que eran cursos secundarios de formación de maestros.

A pesar de la delimitación disciplinaria, es importante reconocer que muchos de los contenidos enseñados en la asignatura de sociología en ese momento provenían de otras ciencias sociales, como la historia y la geografía (Guelfi, 2007). Este escenario se profundizó por el hecho de que los primeros profesores de sociología en Brasil eran autodidactas y no tenían antecedentes académicos en esta área del conocimiento ${ }^{2}$ (Meucci, 2011).

Aún en la década de 1930, se crearon las primeras carreras de pregrado en ciencias sociales ${ }^{3}$, lo que permitió la creación de la primera generación de profesores de sociología en Brasil con formación en el área. Sin embargo, es importante indicar que estos cursos también se centraron en la formación de investigadores y técnicos, y no solo en profesores. Además, la concepción de ciencias sociales en estos cursos era más amplia que hoy en Brasil, incluyendo distintas ciencias, como antropología, ciencias políticas, demografía, geografía, historia, psicología social, sociología, etc.

Es interesante notar que, al mismo tiempo que la sociología comenzó a ganar espacio en la educación superior, perdió espacio en la escuela, ya que la Reforma Capanema (1942) extinguió los cursos complementarios, por lo que la sociología continuó siendo enseñada en la educación secundaria. apenas en las Escuelas Normales.

La sociología como asignatura escolar permaneció ausente del currículum escolar a nivel nacional a lo largo del siglo XX, sin embargo, es posible indicar que sus contenidos fueron incorporados por otras asignaturas, como "Estudios Sociales" y "Organización Social y Política de

\footnotetext{
${ }^{2}$ Una de las pocas excepciones del período fue Gilberto Freyre (1900-1987), quien durante su Maestría en Historia Social en la Universidad de Columbia tomó cursos en el Departamento de Sociología.

${ }^{3}$ Las primeras carreras de pregrado en ciencias sociales en Brasil son las de la Escuela Libre de Sociología y Política de São Paulo (1933), Universidad de São Paulo (1934), Universidad del Distrito Federal (1935), Facultad de Filosofía, Ciencias y Letras de Paraná (1938) y Facultad de Filosofía de Bahía (1941).
} 
Brasil" (OSPB). Este proceso de debilitamiento del carácter disciplinario de la sociología en la educación secundaria se profundiza en la dictadura militar que comenzó en 1964, especialmente con las reformas educativas que tuvieron lugar en la década de 1970. Según Silva (2007, p. 412413):

Nos governos militares, rompe-se com o eixo do ensino baseado nas disciplinas tradicionais das ciências humanas e ciências naturais. O currículo do $1 .^{\circ}$ e $2 .^{\circ}$ grau regionaliza os conhecimentos agrupando-os em áreas de aplicabilidade tecnológica imediata. Assim, o aluno não precisaria mais aprender literatura, mas, sim Comunicação e Expressão, a partir do ensino das regras da gramática. Os alunos não precisariam aprender os fundamentos da Física, a dinâmica, a quântica, mas, somente algumas fórmulas que seriam utilizadas na elaboração de alguma tarefa básica da contabilidade, da construção civil etc. O mesmo valendo para todas as outras disciplinas e áreas de conhecimento. Os livros didáticos demonstram o esvaziamento científico que se oficializou nas escolas. Os estudos por instrução programada, os testes e os exercícios de completar. As Ciências Sociais foram completamente ideologizadas, enfraquecendo a História e a Geografia como disciplinas científicas. Os Estudos Sociais regionalizaram essas ciências em um campo amplo de aplicabilidade. A Educação Moral e Cívica substituiu o que poderia ser o ensino de Filosofia e de Sociologia, jogando fora, séculos de reflexões, no caso da Filosofia e, pelo menos, cento e cinqüenta anos de Sociologia e Antropologia. Esse modelo de currículo é o regionalizado, em outros termos, como estamos acostumados a falar é o currículo tecnicista.

Podemos observar, por lo tanto, que la enseñanza de la sociología en este período encontró numerosos desafíos institucionales y políticos. Al mismo tiempo, los cursos de pregrado y posgrado en ciencias sociales se expandieron durante este período (Liedke Filho, 2005), consolidando la sociología como una ciencia centrada en la investigación y la educación superior. En parte, este escenario puede explicar el bajo interés de los sociólogos brasileños en la enseñanza de la sociología y el mayor prestigio que tiene la licenciatura en relación con la formación de profesores.

Es en el contexto de la redemocratización de la sociedad brasileña a partir de la década de 1980 que la sociología se introduce gradualmente en los planes de estudio escolares en varios estados de Brasil. Debe entenderse que en ese momento la educación llegó a ser entendida como fundamental para la consolidación de un nuevo proyecto democrático para la sociedad brasileña (Weber, 1996), y la sociología también fue percibida como una ciencia que podría contribuir a este proceso. Al principio, son las asociaciones profesionales, como la Federación Nacional de Sociólogos, las que inician un movimiento a favor del retorno de la sociología en la educación secundaria, más que las asociaciones académicas (Moraes, 2011).

La publicación de la Ley de Directrices y Bases de la Educación Nacional (LDBEN) en 1996 reforzó el argumento sobre el regreso de la sociología al currículo escolar, ya que indicaba que los estudiantes egresados de la educación secundaria deberían tener conocimientos de sociología y filosofía para el ejercicio de la ciudadanía. En principio, como lo señalan las Directrices Curriculares Nacionales de la Educación Secundaria de 1998, se interpretó que el conocimiento de la sociología y la filosofía debería enseñarse de manera interdisciplinaria a través de otras ciencias sociales, como la geografía y la historia (Moraes, 2007).

Solo con el parecer del Consejo Nacional de Educación (CNE) N 38/2006, y más tarde con la Ley $N^{\circ} 11.684$ de 2008, esa sociología se ha convertido en una asignatura obligatoria en todos los años de educación secundaria en Brasil. Esta obligación persistió hasta 2017, cuando la Reforma de la Escuela Secundaria eliminó el carácter obligatorio de todas las materias escolares, con la excepción del portugués y las matemáticas, las demás deberían ser indicadas por los estados.

A pesar de estar de acuerdo con Moraes (2011), quien indica que no existe una relación automática entre la presencia de sociología en el currículo escolar y la democracia, ya que esa asignatura estaba presente en períodos autoritarios (como en el Estado Novo en los años de 1930) y 
ausente en períodos democráticos (como en el período después de la redemocratización en los años de 1980) - la hipótesis planteada por Meucci (2015, p. 258) parece plausible:

Considerando que as bases do retorno recente da Sociologia à escola foram o processo de democratização, a crise atual (que, em resumo, duvida da eficácia das instituições democráticas formuladas desde 1988) tem como efeito colocar em questão seu estatuto de conhecimento. Em resumo, lanço a seguinte hipótese: os vínculos da Sociologia escolar com a democratização foram tão fortemente constituídos que o crescimento da percepção sobre o fracasso das conquistas democráticas conduz ao aumento do questionamento acerca da sua posição curricular e sua finalidade escolar. Ou seja, a desqualificação da democracia conduz também à uma 'desqualificação do currículo escolar democrático', em particular da Sociologia, tão recentemente institucionalizada sob o argumento da 'formação cidadã'

Esto significa que el cuestionamiento sobre el lugar de la sociología en la educación secundaria tiende a ampliarse en el momento en que el péndulo de la democracia en Brasil avanza hacia un revés democrático (Avritzer, 2018). Este problema es evidente en los ataques públicos que tuvieron lugar bajo el gobierno de Jair Bolsonaro sobre las humanidades, y sobre la sociología en particular (Blois y Oliveira, 2019). Sin embargo, es importante tener en cuenta que estos procesos son bastante complejos y contradictorios, ya que la Sociología continúa integrando el área de conocimiento de "Ciencias humanas y sus tecnologías" en el Exame Nacional do Ensino Médio ENEM (Examen Nacional de la Educación Secundaria) como un componente curricular.

\section{EXPERIENCIAS EN LA ENSEÑANZa de LA SOCIOlOgía EN BRASIL EN PERÍOdo RECIENTE (2008-2017)}

A pesar del poco tiempo como asignatura obligatoria de educación secundaria, como se demostró en la sección anterior, la enseñanza de la sociología tiene una larga historia en Brasil. En este breve período entre 2008 y 2017 hubo una intensa acumulación de experiencias pedagógicas e institucionales en esta área. Tales experiencias nos permiten pensar sobre los fundamentos de la didáctica en la enseñanza de la sociología, insertándola en una discusión más amplia sobre la DCS.

Un efecto más inmediato que tuvo la reintroducción de la sociología en la educación secundaria fue la expansión del número de cursos de formación inicial de profesores en esta área (Oliveira, 2015). Además de la expansión cuantitativa, también se desarrollaron nuevos modelos de formación, como aquellos con un enfoque más interdisciplinario y una reevaluación de los cursos existentes. Paralelamente a esto, las nuevas directrices para los cursos de formación de profesores en Brasil en 2015 reforzaron el perfil autónomo de estos cursos en relación con las licenciaturas en ciencias sociales/sociología.

A partir de 2009, se ha credo en Brasil el Programa Institucional de Bolsas de Iniciação à Docencia - PIBID (Programa Institucional de Becas de Iniciación a la Enseñanza) con financiación de la Coordenação de Aperfeiçoamento de Pessoal de Ensino Superior - CAPES (Coordinación para el Mejoramiento del Personal de Educación Superior). Este programa, además de garantizar becas para estudiantes universitarios interesados en la formación de profesores, proporcionó una mayor proximidad entre las universidades y las escuelas, ampliando el espacio de práctica docente. En los cursos de formación de profesores de sociología, este programa tuvo un enorme impacto, ya que estos cursos históricamente valoraron más la teoría que la práctica.

En 2012, la sociología se convirtió en parte del Programa Nacional do Livro Didático PNLD (Programa Nacional de Libros de Texto), que es una política de Estado en Brasil que evalúa, compra y distribuye libros de texto a todas las escuelas públicas de Brasil. Se entiende que los libros textos de sociología también deben incluir conocimientos de antropología y ciencias políticas, ya que la formación de los profesores de sociología se lleva a cabo en cursos de ciencias sociales. 
También en 2012, se aprobó el primer máster profesional en enseñanza de sociología, el máster en ciencias sociales en la escuela secundaria en la Fundação Joaquim Nabuco - FUNDAJ (Fundación Joaquim Nabuco). Este máster está dirigido a profesores que enseñan sociología en la educación básica, en muchos casos, profesores con formación inicial en otras ciencias sociales, con el objetivo de contribuir a su calificación profesional (Zarias, Monteiro y Barreto, 2014). En 2018, comenzaron las actividades del máster profesional en enseñanza de sociología en rede, que involucraron a nueve universidades públicas diferentes en Brasil.

A estas acciones se suman avances en el campo académico, como la creación del Grupo de Trabajo sobre enseñanza de sociología en el Congreso Brasileño de Sociología en 2005; el Encuentro nacional de enseñanza de sociología en educación básica (ENESEB) en 2009; de la Asociación Brasileña de Enseñanza de las Ciencias Sociales (ABECS) en 2012.

La eliminación de la sociología como asignatura obligatoria (y otras ciencias sociales) en 2017 en la Reforma de la Escuela Secundaria fue ciertamente un paso atrás, pero la sociología sigue siendo parte del PNLD, así como el ENEM, lo que demuestra su persistencia en el currículum escolar brasileño.

\section{EN BUSCA DE UNA DIDÁCTICA DE LA SOCIOLOGÍA}

Aunque la sociología tiene una larga historia en la educación secundaria, como se demostró en las secciones anteriores, es importante darse cuenta de que esta asignatura ha adquirido nuevos Esignificados en el período reciente. Esto refleja los cambios políticos, sociales y académicos por los cuales atravesó Brasil. El regreso de la sociología a la educación secundaria en 2008 ocurrió en un momento en que esta ciencia estaba bien establecida en las universidades, y el rol de las ciencias humanas y sociales en el currículo escolar se estaba expandiendo. No podemos olvidar que en 2003 se introdujo la enseñanza de la historia y las culturas afrobrasileñas, incorporando en 2008 también la cuestión indígena.

Con respecto a los principios que guían la enseñanza de la sociología, es importante reconocer el rol de las Orientações Curriculares Nacionais do Ensino Médio - OCNEM (Orientaciones Curriculares Nacionales de la Educación Secundaria) publicadas en 2006, que destacan la necesidad de mediaciones pedagógicas para pensar en la recontextualización del conocimiento sociológico para la educación secundaria. Además, la investigación sería un principio pedagógico para la enseñanza de la sociología y la desnaturalización y extrañeza de la vida social serían principios epistemológicos (Brasil, 2006). Otro punto importante que trajo este documento fue la orientación de organizar la enseñanza de la sociología a través de la articulación entre temas, teorías y conceptos, que serían los principios metodológicos de su enseñanza. De acuerdo con esas orientaciones:

Apesar desse contexto, pode-se verificar que pelo menos três tipos de recortes são reiterados nas propostas construídas para o ensino de Sociologia no nível médio e encontráveis nos parâmetros curriculares oficiais, nos livros didáticos e mesmo nas escolas. São eles: conceitos, temas e teorias. A tendência é os professores, os livros e as propostas apresentarem esses recortes separadamente quando não optam por trabalhar somente com um deles. $\mathrm{O}$ que se propõe aqui para a reflexão dos professores é que esses recortes podem ser tomados como mutuamente referentes, isto é, rigorosamente seria impossível trabalhar com um recorte sem se referir aos outros. O que é possível fazer é tomar um deles como "centro" e os outros como referenciais (Silva, 1986). Ao se tomar um conceito- recorte conceitual -, este tanto faz parte da aplicação de um tema quanto tem uma significação específica de acordo com uma teoria, do contrário os conceitos sociológicos seriam apenas um glossário sem sentido, pelo menos para alunos do ensino médio. Um tema não pode ser tratado sem o recurso a conceitos e a teorias sociológicas senão se banaliza, vira senso comum, conversa de botequim. Do mesmo modo, as teorias são compostas por conceitos e ganham concretude quando aplicadas a um tema ou objeto da 
Sociologia, mas a teoria a seco só produz, para esses alunos, desinteresse. Entende-se também que esses recortes se referem às três dimensões necessárias a que deve atender o ensino de Sociologia: uma explicativa ou compreensiva - teorias; uma lingüística ou discursiva conceitos; e uma empírica ou concreta - temas. (Brasil, 2016, p. 116-117).

Aunque las OCNEM no han indicado cuáles son los contenidos de sociología que deberían ser enseñados en la escuela, han producido importantes indicaciones metodológicas. Podríamos afirmar que ese documento es el marco fundamental para la formulación de una didáctica de la Sociología en Brasil. Todavía uno de los mayores desafíos para efectuar esa articulación es la distancia entre la formación del profesorado brasileño en sociología y el currículum de esa asignatura en la escuela. La formación del profesorado está estructurada principalmente a partir de cuestiones teóricas, ya que el currículum está organizado principalmente a partir de temas. Pipkin (2017) apunta que algunas prácticas didácticas de los profesores de sociología parece ser un reflejo de un conjunto de disposiciones específicas adquiridas en la formación sociológica universitaria. De acuerdo con Pipkin (2017):

En el caso del Profesorado de Sociología, los alumnos - pero también los profesores en ejercicio que hemos entrevistado- consideran que enseñar un tema implica enseñar la teoría de algún autor sobre este tema. Por ejemplo, si el profesor del curso donde van a realizar las prácticas les pide que enseñen algún tema vinculado con el sistema político, rápidamente elaboran una planificación para enseñar el análisis de Weber sobre el Estado o el poder. Si se trata de un tema económico, enseñan Marx; sobre Sociología de la cultura, Bourdieu, y así desfilan los diferentes autores que estudiaron en su carrera. (Pipkin, 2017, p. 32).

La articulación entre teorías, conceptos y temas es un ejercicio complejo, obviamente que los estudiantes de Secundaria deben conocer los autores de la sociología, pero los autores y sus teorías no pueden ser los objetivos de la enseñanza de la sociología en la escuela, la finalidad es la comprensión de la realidad social y para eso los autores y sus teorías son herramientas. Para Oliveira (2014) es necesario reconocer la imposibilidad de transposición de los contenidos de la sociología universitaria para la educación secundaria, en términos de contenidos y metodologías; partiéndose de la realidad social de los estudiantes, preguntándoles que saben del mundo social y que buscan saber.

La sociología debe producir una ruptura epistemológica con la realidad mediata (Bourdieu, Chamboredon y Passeron, 2000), de modo que la didáctica de la sociología sea una forma pedagógica de producir esa ruptura. El punto de partida para esa ruptura sería la realidad social de los estudiantes, que ellos saben del mundo social, como explican los fenómenos que observan, pero la idea no es replicar el sentido común, sino demonstrar la complejidad de la realidad. Para Hamlin (2009), el objetivo de la enseñanza de la sociología en la escuela es desnaturalizar el sentido común, percibir que sus explicaciones de la realidad no son la realidad en sí misma. Las categorías sociológicas serían como gafas para ver la realidad, que posibilitan una percepción más elaborada de la realidad.

Las teorías sociológicas, comprendidas como interpretaciones científicas de la realidad, producen distintas categorías que pueden ser utilizadas en la Educación Secundaria. Pero, evidentemente como la sociología retira las certezas que tenemos, no siempre es fácil utilizarlas en nuestras clases. Truong (2019), por ejemplo, cuando enseñaba la teoría de Pierre Bourdieu para estudiantes de la Educación Secundaria ha percibido algunos retos para enseñar sociología. En su experiencia como profesor, Truong relata que los estudiantes han recibido muy bien la teoría de Bourdieu inicialmente, han identificado claramente los esquemas de dominación en sus propias vidas, pero cuando se han adentrado en el debate acerca de las posibilidades de los estudiantes más pobres para avanzar en los estudios formales, han rechazado la teoría de Bourdieu, afirmando que el sociólogo francés está equivocado y que ellos serían una excepción a la regla. 
[...] essa experiência também coloca em evidência a dificuldade própria da sociologia, que é a recepção da objetivação de suas práticas e a configuração de seu próprio percurso social. Isto é, no nosso caso, ainda maior para superar, lança luz sobre a existência de uma barreira social e cultural entre o professor e seus alunos para enfatizar a influência que pode ter no processo de reprodução social, mesmo que a sala de aula deva ser um lugar de vida e um aprendizado coerente onde um único objetivo comum deve animar todos os protagonistas das interações: entender e ter sucesso. E, no contexto em que ensinei, ocorreu-me que qualquer coisa que pudesse prejudicar a coesão do grupo de classe rapidamente levava a um aumento das dificuldades escolares, porque favorece estratégias para evitar encarar o trabalho. (Truong, 2019, p. 290).

Podremos percibir que la sociología parte de la realidad empírica, la explica con las teorías a través de las categorías sociológicas buscando objetivar la realidad. Se para la historia el alejamiento temporal ayuda para una mejor comprensión del tiempo presiente, para la sociología el alejamiento cultural y los datos sociales (estadísticos, surveys, etnografías etc.) tienen ese rol. Esa relación también puede ser puesta, en otros términos:

La enseñanza de las ciencias sociales se dirige al alumnado delas escuelas infantil, primaria y secundaria. Sus prácticas vienen, en parte, determinadas por el currículo y se caracterizan por las relaciones que en ellas se establecen entre el saber social (objeto de enseñanza y de aprendizaje), el alumnado (con sus representaciones previas del saber), y el profesorado (con sus ideas sobre el saber, la enseñanza y el aprendizaje). (Pagès, 2002, p. 262).

La relación entre esas tras partes constituye los fundamentos de la DCS, y por consecuencia también los fundamentos para una didáctica de la sociología, la especificidad principal que hay en una didáctica de la sociológica relacionase con la especificidad de la sociología con relación a las demás ciencias sociales. Para Giddens (1997) aunque las teorías sociales produzcan explicaciones acerca de la realidad social, la sociología es la ciencia especializada en las transformaciones y funcionamiento de las sociedades modernas. La didáctica de la sociología debe buscar una comprensión de las sociedades modernas utilizándose de los fundamentos de la DCS, objetivando la realidad social a partir de las categorías sociológicas.

Para la realización de ese ejercicio de objetivación de la realidad la investigación sociológica sería fundamental para los profesores, ese sería un de los principios de las OCNEM. A pesar de reconocer la importancia de la investigación para la actividad de enseñanza y aprendizaje en todos los niveles, Gomes (2007) señala las dificultades para implementar esta práctica, ya que incluso en la educación superior, se encuentran dificultades y obstáculos para la articulación de estas actividades. Por otro lado, Moares y Guimarães (2010, p. 54) señalan los siguientes problemas:

A pesquisa como pressuposto epistemológico desenvolve no estudante do Ensino Médio a capacidade de observação e crítica: ele percebe, então, uma nova realidade a partir da análise sociológica do que está à sua volta. O impacto do estranhamento, no primeiro momento, pode ser seguido pela satisfação de responder concretamente a perguntas do tipo "para que serve a Sociologia?”

Se puede entender, por lo tanto, que esta sería una investigación adecuada a la educación secundaria, limitada por el contexto de esa realidad. Acerca de la investigación en didáctica de las ciencias sociales Prats (2002) realiza los siguientes consejos:

En los últimos veinte años se ha venido consolidando la idea de que el profesorado que está impartiendo clase, si lo hace con determinada actitud, predisposición, conocimientos y 
objetivos, está realizando una investigación por medio de su acción didáctica. Esta idea es sumamente respetable, aunque suponga un uso (mejor decir abuso) del concepto de investigación que, en absoluto, es homologable a lo que la comunidad científica entiende por ciencia. De hecho, lo que viene denominándose investigación en la acción, desde mi punto de vista, una de las formas más conscientes, educativas y ricas de ejercer la docencia. Otra cosa es qué aportaciones produce este tipo de acción didáctica para, por sí sola, construir un cuerpo teórico, relativamente estable, y para la determinación de las mínimas regularidades que se establecen en cualquier campo de investigación científica de lo social. Ello no supone que cualquier práctica didáctica, la citada quizá en mayor medida, no produzca un apreciable y transmisible conocimiento del proceso de enseñanza y aprendizaje; y, además, que este planteamiento sea la base de posibles y fructíferas investigaciones. Pero no creo que podamos hablar, en sentido riguroso, de una actividad de investigación científica. (Prats, 2002, p. 82).

Partiéndose de esa concepción de investigación en la acción es más fácil percibir la relación entre una didáctica específica para la sociología y su investigación. La investigación sociológica de los profesores de sociología debe ser también una investigación acerca de la didáctica específica presentes en sus prácticas.

Considerando la centralidad que los libros de texto poseen en las escuelas brasileñas también es relevante considerar como la investigación aparece en eses materiales, especialmente porque hay en eses libros un "manual del profesor”, con sugerencias de investigación y actividades. Según Oliveira y Cigales (2015), esta idea aparece relativamente heterogénea en los libros de texto.

Tendencialmente os livros apontaram, em sua maioria, para uma forte presença das pesquisas informativas, dentro da nomenclatura que estamos aqui utilizando, dando um menor destaque para a discussão, e mesmo o ensino, dos fundamentos metodológicos da pesquisa sociológica. Isso pode ocorrer por um conjunto de razões; nesse momento, podemos esboçar algumas hipóteses que poderiam explicar o porquê dessa prática nos livros: (a) tendencialmente os profissionais com formação no campo das Ciências Sociais poderiam perceber o ensino da pesquisa como algo demasiado complexo para os alunos do Ensino Médio, como indica a pesquisa de Santos (2002), de tal modo que os autores também compartilhariam dessa perspectiva, difundindo principalmente uma versão bastante simplificada do fazer da pesquisa sociológica; (b) considerando a realidade do Ensino de Sociologia no Brasil, conduzido de forma esmagadora por professores que não possuem formação no campo das Ciências Sociais, portanto não possuem domínio de seus fundamentos metodológicos, elaborar um livro didático com uma forte ênfase na pesquisa sociológica implicaria a criação de problemas que emergiriam em sala de aula, ou ainda um afastamento desses profissionais com relação ao material didático; (c) trata- -se de uma difícil escolha por parte dos autores, tendo em vista que uma das características metodológicas da Sociologia é sua pluralidade de métodos e técnicas de pesquisa (Martins, 2007; Melo, 2014), de tal modo que seria talvez inviável abordar essa pluralidade de forma relativamente profunda. (Oliveira y Cigales, 2015, p. 287).

Podríamos resumir el problema indicando que los libros de texto reconocen la importancia de la investigación en la práctica pedagógica de sociología, sin embargo, también reconocen los límites objetivos que existen para su realización, lo que refuerza una perspectiva más simplificada de la investigación escolar.

La elaboración de una didáctica de la sociología, por lo tanto, dialoga con el campo más amplio de la DCS, pero también se inserta en el conocimiento acumulado sobre las metodologías de enseñanza de la sociología en la educación secundaria. También debe reforzarse la importancia que existe para la educación secundaria brasileña el ENEM, que tiene una matriz de referencia en relación con las áreas de conocimiento, esta matriz indica las competencias de cada área, así como los objetos de conocimiento.

Las competencias de las humanidades serían: a) Comprender los elementos culturales que constituyen las identidades; b) Comprender las transformaciones de los espacios geográficos como 
producto de las relaciones de poder socioeconómico y cultural; c) Comprender la producción y el papel histórico de las instituciones sociales, políticas y económicas, asociándolas con diferentes grupos, conflictos y movimientos sociales; d) Comprender las transformaciones técnicas y tecnológicas y su impacto en los procesos de producción, el desarrollo del conocimiento y la vida social; e) Usar el conocimiento histórico para comprender y valorar los fundamentos de ciudadanía y democracia, favoreciendo el desempeño consciente del individuo en la sociedad; f) Comprender la sociedad y la naturaleza, reconociendo sus interacciones en el espacio en diferentes contextos históricos y geográficos.

Los objetos de conocimiento de las ciencias humanas serían: a) Diversidad cultural, conflictos y vida en sociedad; b) Formas de organización social, movimientos sociales, pensamiento político y acción del Estado; c) Características y transformaciones de las estructuras productivas; d) Dominios naturales y la relación entre los humanos y el medio ambiente; e) Representación espacial.

A esto se agrega la Base Nacional Comum Curricular - BNCC (Base Nacional Común Curricular) para educación secundaria, publicada en 2018. Según Silva, Alves Neto y Vicente (2015), la propuesta de un plan de estudios nacional se ha señalado desde la constitución de 1988, sin embargo, aún no se ha consolidado hasta entonces. Los autores señalan algunos desafíos para la consolidación de este proyecto:

Um dos desafios é consolidar os procedimentos democráticos e o fortalecimento de esferas públicas de discussões que consigam mobilizar os grupos sociais em torno dos valores dos direitos sociais e dos direitos humanos. A não concordância dos grupos sociais com a validade desses direitos enfraquece as possibilidades da educação pública de qualidade. Assim, as arenas decisórias das definições das ações públicas e das políticas educacionais devem ser valorizadas e potencializadas, especialmente nas dinâmicas das escolas em conexão com as redes municipais, estaduais e nacionais, na busca de consensos sempre provisórios, mas que ajudem a desenvolver as ações e as formações para a democracia. (Silva, Alves Neto y Vicente, 2015, p. 341).

Debe entenderse que el BNCC se desarrolló en diferentes momentos, comenzando su discusión reciente aún bajo el gobierno de Dilma Rousseff, extendiéndose después del golpe político en 2016, y su publicación se produjo solo en 2018, bajo el gobierno de Michel Temer. En este proceso, Miqueli (2020) destaca la participación de múltiples agentes, que tienen intereses diferentes y que participan en la preparación del BNCC. En su versión final, este documento no produjo un plan de estudios nacional, indicando solo pautas más generales, atribuyendo a los estados la tarea de determinar las materias y los contenidos que se enseñarán en la educación secundaria.

Se entiende que la formulación de una didáctica de la sociología implica, por lo tanto, un diálogo con la DCS en general, y la especificidad que tiene la enseñanza de la sociología en Brasil. En este sentido, es importante comprender la especificidad de la DCS.

Por tanto, la especificidad del conocimiento de la DCS deriva de considerar que su objeto de estudio, análisis e investigación abarca todo el proceso que va desde las finalidades que la sociedad y el currículo otorgan a las disciplinas sociales escolares, a la selección del conocimiento pertinente en cada etapa educativa y en cada curso, hasta su conversión en objeto de enseñanza y aprendizaje, proponiendo soluciones adecuadas en cada uno de los estadios del proceso y evaluándolas. (Pagès, 2002, p. 262).

Según Pagès (2002), las didácticas específicas son disciplinas puente, lo que permite la construcción de un campo específico de conocimiento. La didáctica de la sociología dialoga, por lo tanto, con el campo teórico más amplio de la sociología y las ciencias de la educación, y también con la DCS. La sociología comparte con la historia, la geografía y otras ciencias sociales una 
reflexión sobre cómo desarrollar el pensamiento crítico sobre la vida social, que está relacionado con la formación para la ciudadanía (Santisteban y Pagès, 2007).

La didáctica de la sociología, por lo tanto, parte de la investigación sociológica sobre la realidad escolar y los procesos de enseñanza y aprendizaje específicos de esta asignatura, dialogando con diferentes tradiciones disciplinarias. La relación entre conceptos, teorías y temas parece ser el trípode fundamental de la sociología, especialmente considerando la fuerte tradición teórica de esta ciencia, pero que en el contexto escolar debe dialogar con los temas que conforman la vida cotidiana de la vida social, algo mediado pedagógicamente por los conceptos sociológicos.

El debate sobre diversos temas como el género, los movimientos sociales, las relaciones raciales, aunque puede ser objeto de una discusión más amplia en el campo de las ciencias sociales, gana especificidad a partir de las categorías y teorías sociológicas. Como Burke (2012) enfatiza, al pensar en la relación entre historia y sociología, mientras que la historia social enfatiza eventos singulares, la sociología tiende a centrarse en las estructuras sociales. En cualquier caso, las ciencias sociales son áreas que se benefician mutuamente de los diálogos interdisciplinarios.

La tradición teórica de la sociología, que históricamente es una disciplina centrada en teorías y autores, no debe desaparecer en la educación secundaria, sino que debe ser redimensionada. Se entiende que la enseñanza de la sociología en la escuela no debe limitarse a la enseñanza de la historia de la sociología, sino que debe desarrollar de una reflexión sociológica sobre el mundo social.

La reflexión sobre la especificidad del conocimiento sociológico es algo constante en esa ciencia. Giddens (1997) indica que uno de los desafíos de la sociología es explicar una realidad para la cual las personas ya producen explicaciones, distanciarse de lo que ocurre en el campo de las ciencias naturales. Para Baum y May (2010), la sociología todavía está cerca de las otras ciencias sociales, distinguiéndose principalmente del tipo de preguntas que hace. Para estos autores, en sociología, los actores sociales se convierten en objeto de investigación, ya que se los entiende como participantes en una red de interdependencia.

La comprensión de esta red de interdependencia en la educación secundaria se desencadena a través de los temas que se llevan al aula. Cuestiones como el racismo, la xenofobia, la desigualdad social y de género, las diferencias culturales, etc., se entienden no como fenómenos dados, sino como productos de la acción humana en medio de esta red de interdependencias (por algunos llamados estructura social). Por lo tanto, la didáctica de la sociología busca comprender los temas planteados en el aula a la luz de las teorías sociológicas, mediadas pedagógicamente por conceptos sociológicos.

Los problemas socialmente relevantes adquieren relevancia en este proceso (Pagès, 2002), ya que la clase debe ser significativa para los estudiantes, debe ser un espacio para la construcción de significados. En este contexto, la producción sociológica de cada país, cada región, gana protagonismo, ya que se ocupa precisamente de los problemas que se consideran socialmente relevantes en cada realidad.

\section{CONSIDERACIONES FINALES}

Este trabajo tuvo como objetivo contribuir al campo más amplio de DCS a través de la discusión específica de la enseñanza de la sociología. Es posible afirmar que la enseñanza de la sociología en la Educación Secundaria es bastante heterogénea, como podemos ver en las investigaciones realizadas en Argentina (Pereyra, Pontremoli, 2014) y Uruguay (Oliveira, 2019; Mañan, Sabatovich, 2020), países vecinos de Brasil, pero que tienen distintos sistemas educativos y modelos para la formación de profesores de sociología, además de una amplia variación en relación con el espacio que ocupa la sociología en el currículo escolar.

La experiencia acumulada nacionalmente en Brasil desde 2008 nos permite afirmar que hay un conjunto significativo de experiencias, modelos y propuestas pedagógicas que pueden apoyar 
una didáctica específica de la sociología. En este momento, esas experiencias están siendo repensadas, considerando el nuevo contexto socio-político brasileño con la ascensión de Jair Bolsonaro en 2018 y con los distintos ataques a las ciencias sociales. Este gobierno asumió la lucha contra la "ideología de género", el "marxismo cultural" y el "globalismo" ${ }^{45}$ como una prioridad en el campo educativo, algo que impacta de modo directo la enseñanza de la sociología. Aunque el escenario pueda indicar un retroceso para las ciencias sociales, y para la sociología en especial, es importante percibir que:

A pesar del escenario actual, marcado por un ataque abierto a las ciencias sociales, y a la autonomía didáctico-pedagógica de la escuela en general, creo que en estos momentos la enseñanza de las ciencias sociales es aún más necesaria. Las ciencias sociales, comprometidas con la formación de ciudadanos y con un ideal democrático de la sociedad, son una clave fundamental en este proceso. Creo que es a través del conocimiento con base científica que las ciencias sociales pueden lograr su propósito en la escuela. (Oliveira, 2020, p. 220).

Por lo tanto, partir de problemas socialmente relevantes se convierte más que nunca en el desafío, no sólo didáctico sino también político. Para eso, comprendo que es necesario avanzar en el diálogo más amplio con DCS para el desarrollo de una didáctica específica de la sociología, fundamentada en la relación entre teorías, conceptos y temas, objetivando la desnaturalización del mundo social, partiéndose del sentido común, pero avanzando en dirección a las explicaciones especializadas de la sociología.

\section{Referencias}

Avritzer, L. (2018). O pêndulo da democracia: uma análise da crise 2013-2018. Novos Estudos, 37(2), 273-289. DOI: 10.25091/s01013300201800020006

Bauman, Z. y May, T. (2010). Aprendendo a pensar com a sociologia. Rio de Janeiro: Jorge Zahar.

Blois, J. P. y Oliveira, A. (2019). La sociología como profesión. Formación, organización y prácticas de las sociólogas y los sociólogos en um escenario de cambio, Temas Sociológicos, (25), 9-24. Recuperado de http://ediciones.ucsh.cl/ojs/index.php/TSUCSH/article/view/2161/1911 en 10 nov. 2020

Bodart, C. y Cigales, M. P. (2017). Ensino de sociologia no Brasil (1993-2015): um estado da arte na pós-graduação. Revista de Ciências Sociais, 48(2), 256-281. Recuperado de http://www.periodicos.ufc.br/revcienso/article/view/19500 en 10 nov. 2020

Bourdieu, P., Chamboredon, J. y Passeron, J. (2000). O Ofício do Sociólogo: preliminares epistemológicas. Petrópolis: Vozes.

Brasil (2006). Orientações curriculares nacionais do ensino médio: Ciências humanas e suas tecnologias Brasília : Ministério da Educação, Secretaria de Educação Básica.

Burke, P. (2012). História e teoria social. São Paulo: Editora UNESP.

Cesarino, L. (2019). Identidade e representação no bolsonarismo. Revista de Antropologia, 62(3), 530-557, DOI: 10.11606/2179-0892.ra.2019.165232

Engerroff, A. M. B. y Cigales, M. (2017). A constituição da Sociologia no Brasil e o Direito: a formação dos intelectuais. Revista Urutágua, (35), 80-101. DOI: 10.4025/urutágua.v0i35.36651

\footnotetext{
${ }^{4}$ Para Cesarino (2019) estas categorías son significantes vacíos, aunque puedan tener mucha fuerza entre los electores de Jair Bolsonaro, principalmente en las redes sociales.

${ }^{5}$ Globalismo sería un proyecto de un gobierno global, siendo una categoría utilizada por los movimientos populistas conservadores como una crítica a la idea de globalización. Para Olavo de Carvalho, considerado un de los principales intelectuales del gobierno de Jair Bolsonaro, es considerado un de los principales críticos al globalismo.
} 
Giddens, A. (1997). As consequências da modernidade. São Paulo: Editora UNESP.

Gomes, A. L. F. (2007). Notas críticas sobre as Orientações Curriculares Nacionais(OCN’s) para o Ensino de Sociologia no Ensino Médio. Revista Cronos, 8(2), 475-486. Recuperado de https://periodicos.ufrn.br/cronos/article/view/1851/pdf_64 en 10 nov. 2020

Gómez-Carrasco, C. J., López-Facal, R. y Rodríguez-Medina, J. (2019). La investigación en Didáctica de las Ciencias Sociales en revistas españolas de Ciencias de la Educación. Un análisis bibliométrico (2007-2017). Didáctica de las ciencias experimentales y sociales, 37, 67-88. DOI:10.7203/DCES.37.14440

Guelfi, W. P. (2007). O movimento da sociologia como disciplina escolar entre 1925 e 1942: as reformas do secundário e os programas de ensino do Colégio Pedro II. Revista Mediações, 12(1), 11-30. DOI: 10.5433/2176-6665.2007v12n1p11

Hamlin, C. L. (2009). Desenvolvendo uma terceira cultura nas escolas: habitus sociológico, estranhamento e desnaturalização de preconceitos. Tomo, (15), 71-82, DOI: 10.21669/tomo.v0i15.489

Liedke Filho, E. (2005). A Sociologia no Brasil: história, teorias e desafios. Sociologias, (14), 376437. DOI: 10.1590/S1517-45222005000200014

Meucci, S. (2015). Sociologia na educação básica no Brasil: um balanço da experiência remota e recente. Ciências Sociais UNISINOS., 51(3) 251-260. DOI: 10.4013/csu.2015.51.3.02

Michetti, M. (2020). Entre a legitimação e a crítica: as disputas acerca da Base Nacional Comum Curricular. Revista Brasileira de Ciências Sociais, 35(102), 1-19. DOI: $10.1590 / 3510221 / 2020$

Moraes. A. C. (2007). Parecer sobre o Ensino de Filosofia e de Sociologia. Revista Mediações, 12 (1), 239-248. DOI: 10.5433/2176-6665.2007v12n1p239

Moraes, A. C. (2011). Ensino de Sociologia: periodização e campanha pela obrigatoriedade. Cadernos CEDES, 31(85), 359-382. DOI: 10.1590/S0101-32622011000300004

Moraes, A. C. y Guimarães, E. F. (2010). Metodologia de Ensino de Ciências Sociais: relendo as OCEM-Sociologia. En A. C. Moares (Ed.), Sociologia: ensino médio (pp. 45-64). Brasília: Ministério da Educação, Secretaria de Educação Básica.

Mañan, O. y Sabatovich, D. (2020). Los cambios curriculares en la construcción del profesorado de sociología en Uruguay: ¿cambió la concepción de sociología que enseña-mos? Espaço do Currículo, 13(2), 203-218. DOI: 10.22478/ufpb.1983-1579.2020v13n1.51122

Oliveira, A. (2013). Revisitando a história do ensino de sociologia na educação básica. Acta Scientiarum Education. 35(2), 179-189. DOI: 10.4025/actascieduc.v35i2.20222

Oliveira, A. (2014). Os Desafios Teórico-Metodológicos do Ensino de Sociologia no Ensino Médio. Perspectiva, 32(3), 1019-1044. DOI: 10.5007/2175-795X.2014v32n3p1019

Oliveira, A. (2015). Cenários, Tendências e Desafios na Formação de Professores de Ciências Sociais no Brasil. Política \& Sociedade, 14(31), 38-61. DOI: 10.5007/21757984.2015v14n31p39

Oliveira, A. (2019). O Ensino de Sociologia no Uruguai: uma análise a partir das narrativas dos professores. Política \& Sociedade, 18(41), 261-279. DOI: 10.5007/21757984.2019v18n41p261

Oliveira, A. (2020). La enseñanza de las Ciencias Sociales en Brasil hoy. REIDICS: Revista de Investigación en Didáctica de las Ciencias Sociales, 7, 207-222. DOI: 10.17398/25310968.07.207

Oliveira, A. y Cigales, M. P. (2015). A pesquisa como princípio pedagógico no ensino de Sociologia: uma análise a partir dos livros selecionados no PNLD 2015. Ciências Sociais UNISINOS, 51(3), 279-289. DOI: 10.4013/csu.2015.51.3.05

Oliveira, A. y Melchioretto, B. (2020). O ensino de sociologia como tema de pesquisa nas ciências sociais brasileiras. Revista Brasileira de Informações Bibliográficas em Ciências Sociais BIB, 91, 1-26. DOI: 10.17666/bib9102/2020 
Oliveira, A. y Santisteban, A. (2020). O currículo para o ensino de ciências sociais: uma mirada para o mundo Ibero Latino Americano. Espaço do Currículo, 13(2), 138-141. DOI: 10.22478/ufpb.1983-1579.2020v13n1.53443

Pagès, J. (2002). Aprender a enseñar historia y ciencias sociales: el currículo y la didáctica de las ciencias sociales. Pensamiento Educativo, 30(1), 255-269. Recuperado de http://pensamientoeducativo.uc.cl/index.php/pel/article/view/222/467 en 14 nov. 2020

Pereyra, D. y Pontremoli, C. (2014). ¿La Sociología está Pasada de Moda? Una discusión sobre la enseñanza de sociología en la escuela media en Argentina: docentes, estudiantes y propuesta curricular. Educação \& Realidade, 39(1), 139-159. DOI: 10.1590/S217562362014000100009

Pipkin, D. (coord.) (2009). Pensar lo social. Un aporte de la enseñanza de la Sociología para la escuela media. Buenos Aires: La Crujía.

Pipkin, D. (2017). La formación de profesores ¿una cuestión epistemológica? Perspectiva, 35(1), 33-48. DOI: 10.5007/2175-795X.2017v35n1p33

Prats, J. (2002). Hacia una definición de la investigación en didáctica de las ciencias sociales. Enseñanza de las Ciencias Sociales, 1, 81-89. Recuperado de https://www.raco.cat/index.php/EnsenanzaCS/article/view/126132/183031 en 14 nov. 2020

Santisteban, A. y Pagès, J. (2007). La educación democrática de la ciudadanía. Una propuesta conceptual. En R. Ma Ávila Ruiz; J. R. López Atxurra; E. Fernández de Larrea. (eds.), Las competencias profesionales para la enseñanza-aprendizaje de las ciencias sociales ante el reto europeo y la globalización (pp. 353-367). Bilbao: AUPDCS.

Silva, I. F. (2007). A sociologia no ensino médio: os desafios institucionais e epistemológicos para a consolidação da disciplina. Revista Cronos, 8(2), 403-427. Recuperado de https://periodicos.ufrn.br/cronos/article/view/1844/pdf_60 en 10 nov. 2020

Silva, I. F., Alves Neto, H. F y Vicente, D. V. (2015). A proposta da Base Nacional Comum Curricular e o debate entre 1988 e 2015. Ciências Sociais UNISINOS, 51(3), 331-342. DOI: 10.4013/csu.2015.51.3.10

Truong, F. (2019). Ensinar Pierre Bourdieu no 9-3: o que falar quer dizer. Política \& Sociedade, 18(41), 280-291. DOI: 10.5007/2175-7984.2019v18n41p280

Villas Bôas, G. (2006). Mudança provocada: passado e futuro no pensamento sociológico brasileiro. Rio de Janeiro: Editora FGV.

Weber, S. (1996). O professorado e o papel da educação na sociedade. Campinas: Papirus.

Zarias, A., Monteiro, A. y Barreto, T. V. (2014). Mestrado Profissional em Ciências Sociais para o Ensino Médio: a experiência nos horizontes da formação continuada para professores. Revista Brasileira de Sociologia, 2(3), 129-152. DOI: 10.20336/rbs.66

Oliveira, A. (2021). En busca de una didáctica de la sociología: aportes desde la educación secundaria brasileña. Didáctica de las ciencias experimentales y sociales, 40, 21-34. DOI: 10.7203/DCES.40.17651. 